\title{
X.
}

\section{Ueber die durch Tuberculose der nächsten Blutsverwandten geschaffene Disposition zu Ohrerkrankungen bei Kindern.}

(Auf Grund meiner Schuluntersuchungen im Kreise Marburg.)

Von

Prof. 0stmann, Marburg a. L.

Im Veriaufe meiner Schuluntersuehungen im Kreise Marburg ${ }^{1}$ ) gewann ieh mehr und mehr den Eindruek, dass nicht wenige von den ausserordentlich zahlreichen Ohrenkrankheiten unter den Volkssohulkindern in einem gewissen Zusammenhang mit der in Oberhessen gleichfalls ungewöhnlich verbreiteten Tuberculose stehen dürften.

Ioh habe deshalb diesem Punkte meine besondere Aufmerksamkeit zugewandt und zunächst für 1679 schwerhörige Volksschulkinder des Kreises festgestellt, ob bezw. wie viele von ihnen Familien entstammen, in denen unter den näehsten Blutsverwandten der Kinder Todesfälle an Tuberenlose vorgekommen sind. Als näehste Blutsverwandte wurden bezeichnet: Grosseltern, Eltern, Onkel, Tanten, Gesehwister.

Meine Vermuthung wurde dureh das Ergebniss der Sammelforsehung, bei der ich in dankenswerthester Weise von den Lehrern des Kreises unterstützt worden bin, in hohem Maasse verstärkt; denn die Zahl der aus tubereulös belasteten Familien 2) stammenden sehwerhörigen Kinder war eine überrasehend grosse, wenn auch für die einzelnen Ortsehaften procentuariseh erheblich wechselnde.

Um nun aber mehr als eine Vermuthung hinsichtlieh des inneren Zusammenhanges zwisehen der Tubereulose der näebsten Blutsverwandten und den Ohrerkrankungen der zugehörigen

1) Dieses Archiv, Bd. 54 .

2) Ich bezeichne in dieser Arbeit als tuberculös belastete Familien solche, in denen Todesfälle an Tuberculose bei Eltern, Grosseltern, Onkel, Tanten oder Geschwistern vorgekommen sind. 
Kinder aussprechen zu können, musste die Gegenprobe gemacht werden, indem festgestellt wurde, wie viele von den $\mathrm{n}$ or mal hörenden Kindern tubereulös belasteten Familien angehörten. ${ }^{1}$ )

Bei der sehr grossen Zahl dieser - 5395 - musste ich auf eine Beantwortung dieser Frage für alle verzichten; schon aus dem Grunde, weil ich nicht erwarten konnte, dass diese erneute, sehr bedeutende Arbeit von allen Lehrern würde übernommen werden. Ich habe mich deshalb nur an die Lehrer gewandt, von denen ich wusste, dass sie ein lebhaftes Interesse an diesen Untersuchungen gewonnen batten und zumeist, Dank ihrer langjährigen Thätigkeit in der Ortschaft, mit den persönliehen Verbältnissen der Kinder gut vertraut waren.

Die Erhebungen sind somit auf nachstehende acht, räumlich zum Theil weit auseinander liegende Landgemeinden: Wollmar, Weitershausen, Michelbach, Amönau, Bürgeln, Niederwetter, Wenkbach und Roth beschränkt worden; doch ist meines Erachtens trotz dieser nothwendigen Beschränkung eine noch hinreichend umfangreiche und dabei möglichst sichere Unterlage für die nachstehenden Ausführungen gewonnen worden.

Die Tabelle I fasst das Ergebniss der Untersuchungen und Erhebungen für jedes der 8 Dörfer zusammen. (S. Tabelle I S. 74f.)

In den 8 Landgemeinden wurden 676 Kinder vom 5 . bis 13. Lebensjahr untersucht; $162=23,9$ Proe. derselben waren schwerhörig, d. h. hörten auf einem oder beiden Ohren nur auf etwa ein Drittel der normalen Entfernung oder weniger.

Diese 676 Kinder gebörten 375 Familien an, von denen 251 Familien (Gruppe a) ${ }^{2}$ ) nu r normalhörende (404) Kinder,

$70=(\text { Gruppe b })^{2}$ ) normalhörende (110) und schwerhörige (82) Kinder, $\left.64=(\text { Gruppe })^{2}\right)^{2}$ n ur schwerbörige (80) Kinder hatten.

Unter den Familien der einzelnen Gruppen waren tuberculös belastet:

Von Gruppe a $69=25,8$ Proc. mit 119 normalhörenden Kindern,

$=\quad=\quad \mathrm{b} 33=49,5$ Proc. mit 34 normalhörenden und 37 schwerhörigen Kindern,

$=\quad=\quad$ c $41=73,4$ Proc. mit 52 schwerhörigen Kindern.

1) Als weitere Ergänzung habe ich die Fragestellung darauf ausgedehnt, wie viele schwerbörige Kinder nicht tuberculös belasteten Familien entstammen. Ueber das Ergebniss dieser Untersuchungen wird unter Erweiterung der hier mitgetheilten an anderer Stelle berichtet werden.

2) Die Bezeichnung als Gruppe a, b, c für die wie oben charakterisirten Familiengruppen werde ich der Vereinfachung halber im weiteren Verlauf der Arbeit stets anwenden. 


\begin{tabular}{|c|c|c|c|c|}
\hline 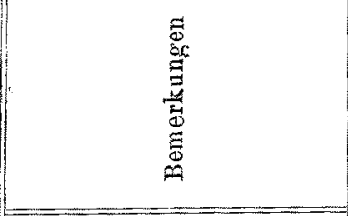 & & & & 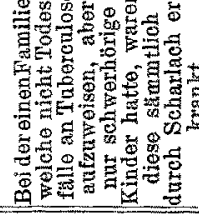 \\
\hline$I I: Z I$ บ0A zq\&\&quəəoxd & 8 & $\underline{8}$ & 8 & 象 \\
\hline 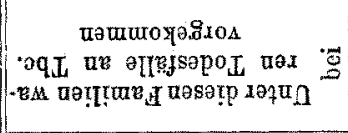 & $\begin{array}{r}0 \\
00 \\
\vdots\end{array}$ & 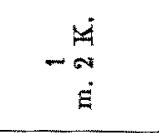 & 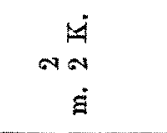 & $\begin{array}{r}10 \\
200 \\
0\end{array}$ \\
\hline 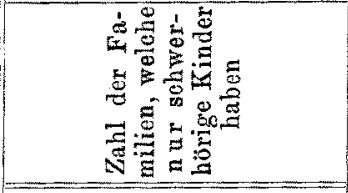 & 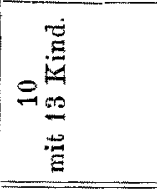 & 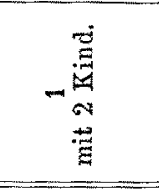 & 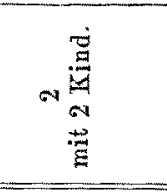 & 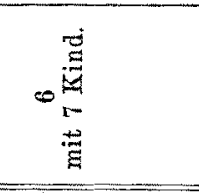 \\
\hline 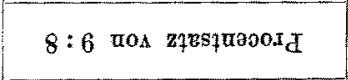 & $=$ & 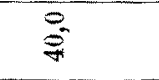 & 8 & $\therefore$ \\
\hline 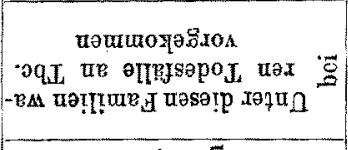 & 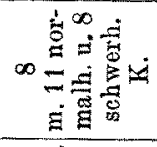 & 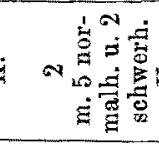 & 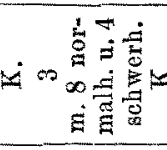 & 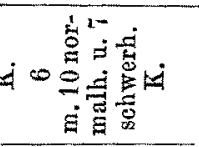 \\
\hline 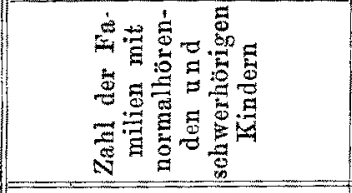 & 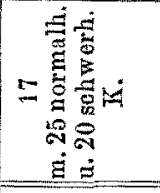 & 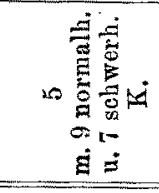 & 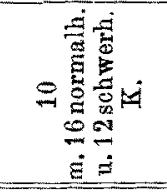 & 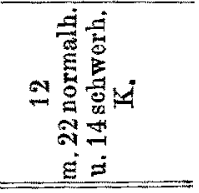 \\
\hline c: 9 u0s zqesquəooxd & 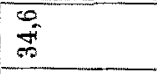 & $\stackrel{8}{ \pm}$ & 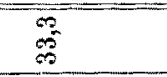 & 8 \\
\hline 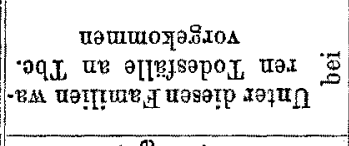 & $\underset{\square}{\stackrel{5}{5}}$ & 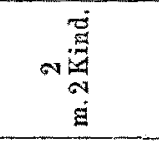 & 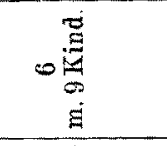 & $\begin{array}{r}\text { คี } \\
\text { ลิ } \\
\dot{8} \\
\end{array}$ \\
\hline 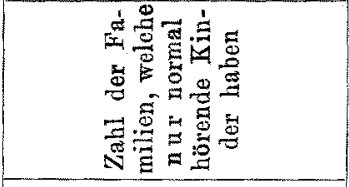 & 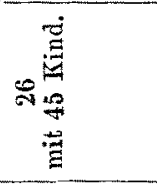 & 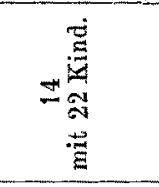 & 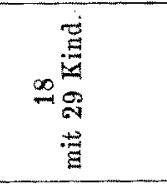 & 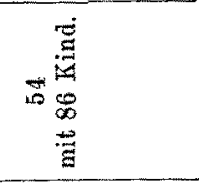 \\
\hline 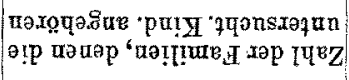 & 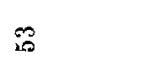 & 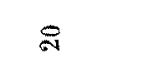 & 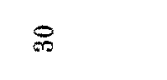 & $\underset{1}{\mathfrak{1}}$ \\
\hline 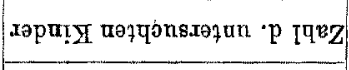 & $\stackrel{8}{9}$ & F & 8 & 丞 \\
\hline 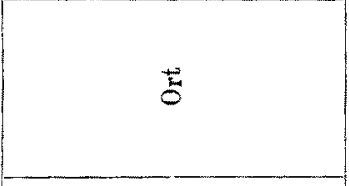 & 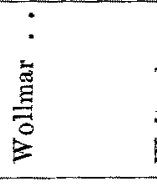 & 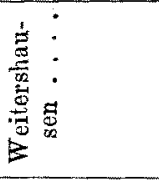 & 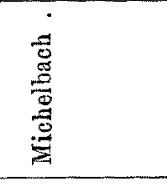 & $\begin{array}{l}\vdots \\
0 \\
0\end{array}$ \\
\hline әриәر & & & $m$ & \\
\hline
\end{tabular}




\begin{tabular}{|c|c|c|c|c|}
\hline 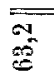 & $\stackrel{8}{8}$ & 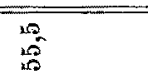 & 商 & $\vec{F}$ \\
\hline 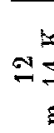 & 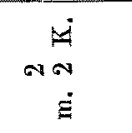 & 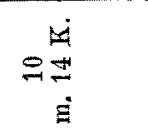 & $\begin{aligned} \dot{b} \\
\dot{g} \\
\dot{g}\end{aligned}$ & 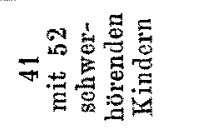 \\
\hline 2 & 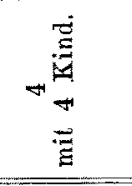 & 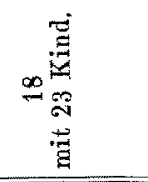 & 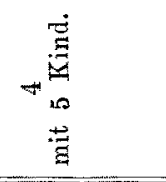 & 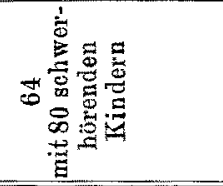 \\
\hline ثิ & : & 今 & 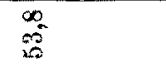 & $\stackrel{80}{9}$ \\
\hline & 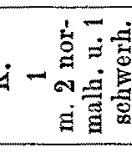 & 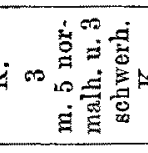 & 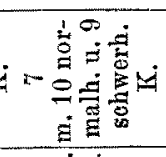 & 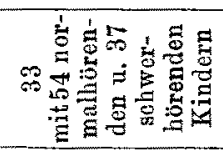 \\
\hline & 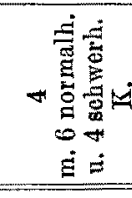 & 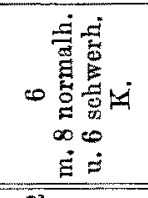 & 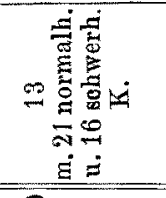 & 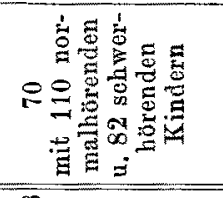 \\
\hline$=$ & $\overline{\text { จิ }}$ & s- & a & 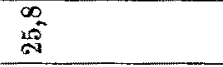 \\
\hline 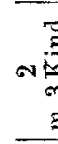 & 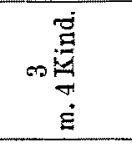 & 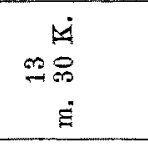 & 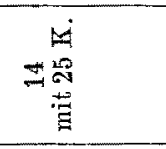 & 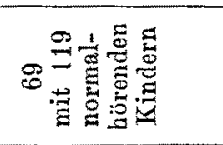 \\
\hline 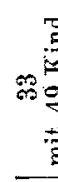 & 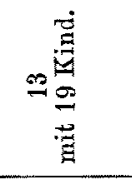 & 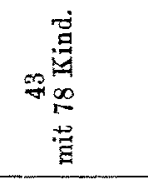 & 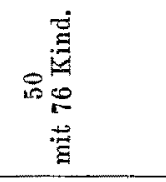 & 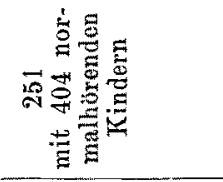 \\
\hline 活 & $\overline{\mathrm{N}}$ & 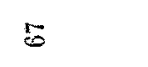 & 8 & $\stackrel{\infty}{\infty}$ \\
\hline 9 & $\mathscr{m}$ & $\stackrel{P}{=}$ & $\stackrel{\infty}{=}$ & 8 \\
\hline 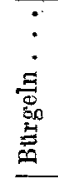 & 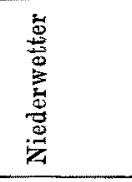 & 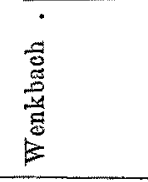 & \begin{tabular}{c}
$\vdots$ \\
$\vdots$ \\
$\overline{3}$ \\
\multirow{3}{*}{}
\end{tabular} & 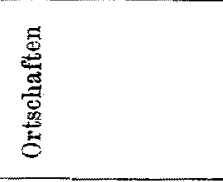 \\
\hline
\end{tabular}


Der Zusammenstellung entnehmen wir als erstes Ergebniss:

Unter denjenigen Familien, welche die relativ meisten schwerhörigen Kinder haben, findet sich auch relativ am häufigsten tubereulöse Belastung der Kinder.

Diese Thatsache machte es an sich sehr wahrscheinlich, dass zwischen der Tubereulose der nächsten Blutsverwandten in aufsteigender Linie und den Ohrerkrankungen der diesen tuberculös belasteten Familien angehörenden Kinder ein, wenn auch nur indirecter ursächlicher Zusammenhang besteht. Ist dies thatsächlich der Fall, so muss angenommen werden, dass dieser $\mathrm{Zu-}$ sammenhang um so sohärfer hervortritt, je näher die an Tuberculose verstorbenen Familienmitglieder dem ohrenkranken Kinde verwandtschaftlich standen, d. h. mit andern Worten: es muss erwartet werden, dass bei Tubereulose der Eltern und Grosseltern eine stärkere Disposition der Kinder zu Ohrerkrankungen im Allgemeinen hervortritt, als bei Tubereulose von Onkel, Tanten und Geschwistern.

In Tabelle II (S. 77) habe ich nach dieser Richtung hin die Ergebnisse der Erhebungen über die tubereulös belasteten Familien der 8 Ortschaften zusammengestellt, und zwar enthält die Tabelle einerseits die 33 tuberculös belasteten Familien der Gruppe b, andererseits die 41 tuberculös belasteten Familien der Gruppe c.

Zur Erläuterung der Tabelle bemerke ich, dass, sofern mehrere Todesfälle an Tubereulose in einer Familie vorgekommen waren, stets der dem Kinde nächststehende Blutsverwandte für die Einreihung in die Rubriken maassgebend war, so dass z. B. beim Tode des Vaters und eines Onkels an Tuberculose nur der Vater in Betracht gezogen wurde u. s. w. (S. Tab. II S. 77.)

Die Tabelle ergiebt:

Todesfälle an Tuberculose in directer, aufsteigender Linie (Eltern und Grosseltern) waren vorgekommen unter den tuberculös belasteten Familien der:

Gruppe b bei $20=60,5$ Proc. der 33 Familien,

$$
=\mathbf{c}=30=73,1==41=
$$

Die schwerhörigen Kinđer der tuberculös belasteten Familien der Gruppe e sind somit relativ sehwerer tubereulös belastet, als die Kinder der tuberculös belasteten Familien der Gruppe b.

Der relativ sehwereren tuberculösen Belastung entspricht also anch hier wiederum das relativ häufigere Auftreten von Ohrenkrankheiten bei den belasteten Kindern. 
Die durch Tuberculose d. Blutsverwandten geschaffene Disposition u. s. w. 77

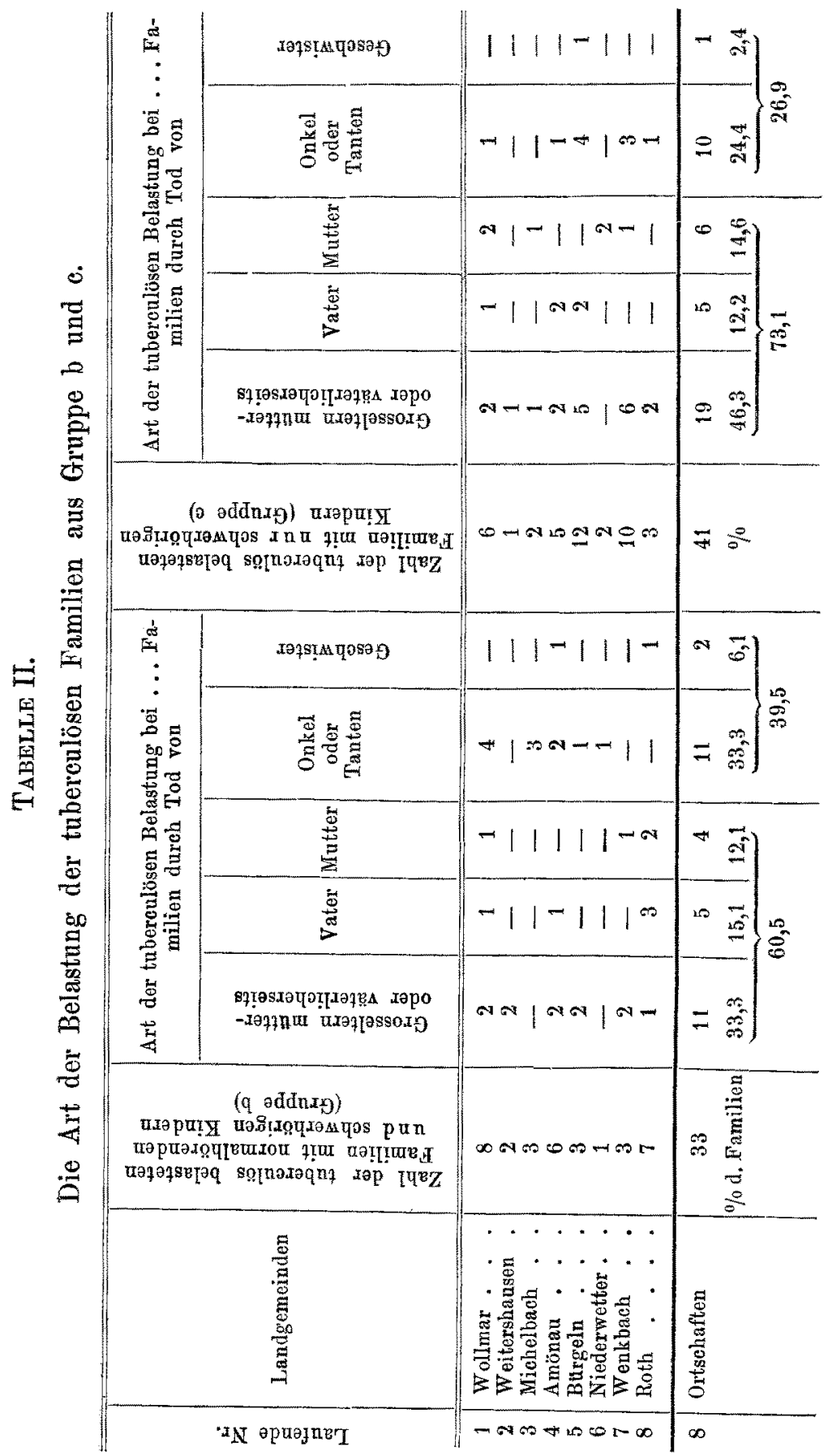


Die Sehlussfolgerung, die wir aus der Annahme eines inneren Zusammenhanges zwischen der Tubereulose der nächsten Blutsverwandten und den Ohrerkrankungen der ihnen zugehörigen Kinder ziehen mussten, hat sich somit dureh die Thatsachen als richtig herausgestellt, wodurch umgekehrt die Richtigkeit der Annahme selbst wesentlich gestützt wird.

Wir können demnach als weiteres Ergebniss feststellen:

Unter den tuberculös belasteten Familien findet sich bei denjenigen, welche die relativ grösste Zahl schwerhöriger Kinder haben, auch relativ am bäufigsten die sehwerste Form der tuberculösen Belastung des Kindes.

Wir können den geheimnissvollen Faden zwischen tuberculöser Belastung des Kindes und Entstehung von Ohrenkrankheiten noch weiter spinnen.

Bei den 89 schwerhörigen Kindern aus tuberculös belasteten Familien der 8 Ortsehaften waren 118 Gehörorgane erkrankt, und zwar wurde bei:

6 Erkranknng des äusseren Ohres;

49 Einziehung des Trommelfells mit und ohne Verkalkung und Trübung;

19 Trübung, Glanzlosigkeit zumeist mit geringer Einziehung;

1 acute Entzündung;

9 chronische Eiterung;

15 kein krankhafter Trommelfellbefund;

19 Narbe oder umschriebene Atrophie des Trommelfells festgestellt.

Die Hörprüfung ergab für:

39 Gebörorgane eine Hörschärfe v. $0-4 \mathrm{~m}$ für zugeflüsterte Zahlen ₹. 1-100

$79=0=4-8 \mathrm{~m}=\quad=\quad=1-100$

Stellt man das Frgebniss der Hörpriffung dieser Gehörorgane dem Gesammtergebniss der Hörprüfungen bei den Schuluntersuchungen gegenü̈ber, so ergiebt sich:

Bei den Schuluntersuchungen wurden von den insgesammt untersuchten 15074 Gehörorganen 2922 schwerhörig befunden.

Von diesen 2922 Gehörorganen hörten:

$$
\begin{aligned}
770 & =26,4 \text { Proc. } 0-4 \mathrm{~m} ; 2152=73,6 \text { Proc. } 4-8 \mathrm{~m}, \\
& \text { Von den } 118 \text { Gehörorganen hörten: } \\
39 & =33,0 \text { Proc. } 0-4 \mathrm{~m} ; \quad 79=67,0 \text { Proc. } 4-8 \mathrm{~m} .
\end{aligned}
$$

Wenn die Zahlen auch sehr versehieden gross sind, so dürfte doch auch hier die ungünstigere Stellung der belasteten Kinder gegenüber dem Durehschnitt aller schwerhörigen Volksschulkinder des Kreises nicht auf reiner Zufälligkeit beruhen, um so mehr als sich des Weiteren zeigen lässt, dass unter den belaste- 
ten Kindern wieder die am schwersten belasteten procentuarisch die meisten erheblich sehwerhörigen stellen.

Zur Führung dieses Nachweises habe ich aus den 8 Dörfern die schwerhörigen Kinder aus den tuberculös belasteten Familien einerseits der Gruppe b, andererseits der Gruppe e nach dem Grade der Schwerhörigkeit der bei ihnen erkrankten Gehörorgane zusammengestellt. Tabelle III bringt die auf die einzelnen Dörfer entfallenden Zahlen.

TABELLE IIT.

\begin{tabular}{|c|c|c|c|c|c|c|c|}
\hline \multirow{2}{*}{ 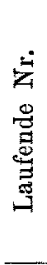 } & \multirow[t]{2}{*}{ Ort } & \multirow{2}{*}{ 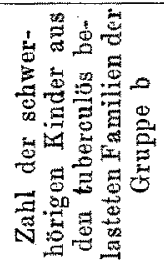 } & \multicolumn{2}{|c|}{$\begin{array}{l}\text { Es hörten von } \\
\text { den erkrank- } \\
\text { ten Gehör- } \\
\text { organen. }\end{array}$} & \multirow{2}{*}{ 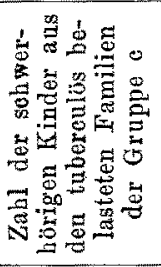 } & \multicolumn{2}{|c|}{$\begin{array}{c}\text { Es börten ron } \\
\text { den erkrank- } \\
\text { ten Gehör- } \\
\text { organen }\end{array}$} \\
\hline & & & $0-4 \mathrm{~m}$ & $4-8 \mathrm{~m}$ & & $0-4$ in & $1-8 m$ \\
\hline 1 & Wollmar & 9 & 2 & 10 & 8 & 5 & 5 \\
\hline 2 & Weitershausen & 2 & - & 2 & 2 & - & 2 \\
\hline 3 & Micbelbach. & 4 & 1 & 5 & 2 & 4 & - \\
\hline 4 & Amönau . & 7 & 2 & 6 & 5 & 5 & 4 \\
\hline$\tilde{a}$ & Burgeln. & 3 & - & 4 & 14 & 4 & 12 \\
\hline 6 & Niedcrwetter & 1 & - & 1 & 2 & 1 & 2 \\
\hline 7 & Wenkbach . & 3 & 1 & 2 & 14 & 8 & 13 \\
\hline 8 & Roth . . . & 9 & 6 & 5 & 4 & - & 6 \\
\hline & & $\mathrm{ma} \quad \overrightarrow{3} 8$ & 12 & 35 & 51 & 27 & 44 \\
\hline
\end{tabular}

Das Ergebniss der Zusammenstellung ist:

die 38 Kinder der Gruppe b hatten 47 erkrankte Gehörorgane $=61,8$ Proc. der 76 unters. Gehörorgane,

die 51 Kinder der Gruppe $c$ hatten 71 erkrankte Gehörorgane $=69,6$ Proc. der 102 unters. Gebörorgane.

Somit weisen die Kinder, welche den erwiesenermaassen durchschnittlich stärker tubereulös belasteten Familien der Gruppe e angehören, procentuarisch auch die grössere Zahl von erkrankten Gehörorganen auf; und nicht allein dies, die erkrankten Gehörorgane zeigen auch relativ hänfiger eine erhebliche Hörstörung, denn von 47 erkrankt. Gehörorganen d. Gruppe b hörten $12=25,5$ Proc. $0-4 \mathrm{~m}$; $35=74,5$ Proc. $4-8 \mathrm{~m}$; $=71=\quad=\quad=\mathrm{c}=27=38,0$ Proc. $0-4 \mathrm{~m} ;$

Das heisst nichts anderes als: $44=62,0$ Proc. $4-8 \mathrm{~m}$.

Die tuberculöse Belastang fördert die Entstehung und but einen ungüntigen Einfluss auf den Ablauf der entstandenen Ohrenkrankbeit aus und zwar um so mehr, je sehwerer die Belastung ist. 
Wenn man bedenkt, dass die Ohrerkrankungen der tuberculös belasteten Kinder nicht tuberculös waren ${ }^{1}$, und weiter, dass auch die Kinder keine sichtbaren Zeichen von Tubereulose boten, so muss die deutlich zu Tage getretene enge Beziehung zwischen tuberculöser Belastung und dem gehäuften Auftreten von Ohrenkrankheiten durch ein von der Tuberculose der nächsten Blutsverwandten ausgehendes und bei dem Kinde die Entstehung von Ohrenkrankheiten fồrderndes Bindeglied erklärt werden.

Ich finde dieses Bindeglied in der erhöhten Vulnerabilität der Schleimhäute der oberen Luftwege sowie in der geringeren Widerstandskraft des Organismus dieser Kinder gegenüber schädigenden Einflüssen.

Bei den aus tuberculös belasteten Familien stammenden Kindern zeigen die Schleimhäute der oberen Luftwege, der Nase, des Rachens, des Kehlkopfes und der Bronehien sehr häufig eine auffallende Reizbarkeit, so dass sie einerseits leicht katarrhalisch erkranken, andererseits, einmal erkrankt, sehwerer abheilen. Insbesondere scheint das adenoide Gewebe der Rachenschleimhaut zur Hypertrophie und Hyperplasie bei diesen Kindern geneigt.

Auch bei der Abheilung kleiner operativer Eingriffe in der Nase - wie galvanokaustische Aetzung der Schleimhaut und Abtragung störender Schwellungen - ist es mir zu wiederholten Malen aufgefallen, wie ungleich bezitglich der nachfolgenden Reaction und Schnelligkeit der endgültigen Abheilung anscheinend ganz gleichartige Fälle verlaufen, und wenn man dem Grunde dieser anscheinend unerklärlichen Ungleichbeit nachging, zeigte sich sehr häufig, dass die Kinder mit übermässig starker Reaction und langsamer Abheilung belastet, und zwar vornehmlich tuberculös belastet waren.

Unter den 89 schwerhörigen Kindern aus tuberculös belasteten Familien unserer 8 Dorfgemeinden waren 22, also $1 / 4$ ausgesprochene Mundathmer und hatten sehr starke Katarrhe der Nase und des Rachens mit Schwellung des adenoiden Gewebes.

Eine grössere Zahl der übrigen hatte gleichfalls selbst in den ausnahmsweise schönen Sommermonaten Katarrhe der Nase und des Rachens, und bei Vielen wurde mir bestätigt, dass sie zu Zeiten der Steigerung der Katarrhe Mundathmer seien.

1) Nur für die eine oder andere der 9 chronischen Eiterungen könnte ein Zweifel bestehen. 
Die durch Tuberculose d. Blutsverwandten geschaffene Disposition $u_{n}$ s. . $\quad 81$

Wir wissen, dass diese ehronisehen Katarrhe der Schleimhäute der oberen Luftwege der Tubereuloseinfection den Boden bereiten, und da bei den sehr beengten Wohnungsverbältnissen auf dem Lande, dem Schmutz in und ansser dem Hause, dem oft völlig unhygienischen Verhaiten tubereulös erkrankter Familienmitglieder die Gelegenheit zur Infection für das Kind in ausgiebigstem Maasse gegeben ist, so kann es nicht Wunder nehmen, dass die Tubereulose sich in so erschreckender Weise von Generation auf Generation fortpflanzt.

Nun sehen wir, dass die Tuberculose der nächsten Blntsrerwandten für das Kind noch andere Folgen hat, die anscheinend weit vom Gebiet der Tubereulose als solchem abliegen.

Das Bindeglied zwischen der Tuberculose der nächsten Blutsverwandten und der Neigung der tuberenlös belasteten Kinder zu Erkrankungen des Schallleitungsapparates muss in der erhöhten Reizbarkeit der Schleimhäute der oberen Luftwege, insbesondere der Nase und des Rachens, gesucht werden, und die weitere Thatsache, dass bei ihnen die Ohrerkrankungen durchsehnittlich zu einer erheblicheren Hörstörung fübren, als dies im Allgemeinen bei gleichartigen Erkrankungen der Fall ist, dürfte sich nur dadurch erklären lassen, dass ihr Organismus eine geringere Widerstandskraft gegen schädigende Einflüsse besitzt.

Diese Thatsachen zeigen den richtigen Weg für die Behandlung dieser Kinder. Neben der localen Behandlung der Ohrerkrankung ist Gesundung und Verminderung der Vulnerabilität der Schleimhaut der oberen Luftwege neben allgemeiner Kräftigung des Organismus durch zweekentsprechende Ernährung, körperliche Uebung, Land- und Seeaufenthalt wie andere zweckentsprechende Maassnahmen, unter denen eine milde Hydrotherapie wohl in Betracht zn ziehen sein würde, anzustreben. Die Kinder für längere Zeit dem elterlichen Hause und damit der unmittelbaren Gefahr der Tubereuloseinfection zu entziehen, wird wohl für die Meisten ein unerfullbarer Wunseh bleiben. 